\title{
ON THE THEORY OF DIOPHANTINE APPROXIMATIONS. II (INHOMOGENEOUS PROBLEMS)
}

\author{
By \\ VERA T. SÓS (Budapest) \\ (Presented by A. RénYl)
}

\section{$\S 1$}

The one-dimensional homogeneous problems of diophantine approximations have a unified treatment by the algorithm of continued fractions. It is possible to give such a geometrical interpretation of convergents and by-denominators (Nebennenner) of continued fractions, which can be extended for the inhomogeneous case, and so it furnishes a parallel treatment of these cases. In this way, e.g., it is possible to prove some simple theorems of Borel type for the inhomogeneous case, to get new lower and upper bounds for the Khintchine constant $c$ defined by

$$
c=\inf _{a} \sup _{\beta} \inf _{x>0, y \text { integers }} x|\alpha x-\beta-y| .
$$

This last result will be treated in [5] and [6].

A similar algorithm, as we give in this paper for the inhomogeneous case, is given in an arithmetical way by J. W. S. CASSELS [1] and used also by R. Descombes [2]. A comparison of both treatments is made in footnote ${ }^{8}$. In $\S 2$ we give this geometrical interpretation of continued fractions for an irrational $a$ and the corresponding algorithm for the inhomogeneous case giving a sequence of multipla $s_{\nu}(\beta)$ which corresponds to the sequence of convergents and by-denominators of continued fractions, and further a sequence of pairs of multipla $q_{k}(\beta), q_{k}^{\prime}(\beta)$ which is a subsequence of $s_{\nu}(\beta)$ and corresponds to the sequence of convergents $q_{k}$ of $\alpha$.

In $\S 3$ we give the proof of some simple theorems of Borel type corresponding to the inhomogeneous case. We call these theorems Borel type, since BOREL sharpened HuRwITZ's theorem to the effect that the inequality

$$
x|x \alpha-y|<\frac{1}{\sqrt{5}}
$$

is soluble with $x$ being among any three consecutive convergents of $\alpha$. It is well known that if we consider two consecutive convergents of $a$, we may assert only the solubility of the inequality

$$
x|x<-y|<\frac{1}{2}
$$


among them, and the constant $\frac{1}{2}$ cannot be diminished. For any convergent one may assert only the inequality

$$
x|x \alpha-y|<1
$$

and again the constant 1 is best-possible. HuRwitz's theorem gives at once that for more than three consecutive convergents generally no inequality better than (1.1) can be proved.

Corresponding to (1.3) and what has been said above we shall prove for the inhomogeneous case that to an irrational $\alpha$ and real $\beta$ the inequality

$$
x|x \alpha-\beta-y|<\frac{2}{3}
$$

is soluble among any pairs $q_{k}(\beta), q_{k}^{\prime}(\beta)$ corresponding to $\alpha$, and the constant $\frac{2}{3}$ is best-possible. Corresponding to $(1.2)$ we shall prove that the inequality

$$
x|x \alpha-\beta-y|<\frac{1}{2}
$$

has a solution among any two consecutive pairs $q_{k}(\beta), q_{k}^{\prime}(\beta), q_{k+1}(\beta), q_{k+1}^{\prime}(\beta)$ corresponding to $\alpha$ and again $\frac{1}{2}$ is best-possible.

Concerning (1.1) we remark first that a theorem corresponding to HuRwitz's is due to CASSELS [1] and asserts that for any real irrational $\alpha$ and $\beta \neq\langle n \alpha\rangle^{1}$ the inequality

$$
x|x \alpha-\beta-\gamma|<\frac{27}{28} \frac{1}{\sqrt{7}}+\varepsilon
$$

has infinitely many solutions (with arbitrary $\varepsilon>0$ ) and the constant $\frac{27}{28} \frac{1}{\sqrt{7}}$ is best-possible. So one would expect that for any real irrational $\alpha$ and $\beta \neq\langle n \alpha\rangle$ the inequality is soluble among any three consecutive pairs $q_{k}(\beta)$, $q_{k}^{\prime}(\beta), q_{k+1}(\beta), q_{k+1}^{\prime}(\beta), q_{k+2}(\beta), q_{k+2}^{\prime}(\beta)$ corresponding to $\alpha$. It is somewhat surprising, after what has been said above, that this is not the case. Moreover, one can show that the number tnree cannot be replaced by any universal constant $l$. For any prescribed positive integer $l$ we shall even show that the inequality

$$
x|x a-\beta-y|<c
$$

with any $c<\frac{1}{\sqrt{5}}$ is not soluble in general among any $l$ consecutive pairs $q_{k+1}(\beta), q_{k+1}^{\prime}(\beta), \ldots, q_{k+l}(\beta), q_{k+2}^{\prime}(\beta)$ of our algorithm.

$1\langle x\rangle$ denotes the fractional part of the real number $x$. 
In what follows let $a$ be irrational, $0<\alpha<1$ and $0 \leqq \beta<1$. Starting in positive direction from a periphery-point $O$ of the circle $K$ with unity periphery, we put up the arcs with length $\beta$ and $n \alpha$ for $n=1,2, \ldots$ We call the endpoints of these as the " $\beta$ " and " $n \alpha$-points", respectively. As it is easy to see, the structure of the $n \alpha$-points for $n=1,2, \ldots$ has the following

PROPERTY A. The directed distance between the $m \alpha$ - and $n \alpha$-points for $m>n$ is the same as that between the $(m-n) c$-point and the point $O$.

DEFinition. We call the $s \propto$-point and the corresponding multipla $s$ adjacent to $\beta$ (corresponding to $\alpha$ ) if there are no $n c$-points with $0<n<s$ in at least one of the two arcs determined by $\beta$ and the $s \alpha$-point.

We shall use the following notations:

The sequence of the adjacent multipla $s$ to $\beta$ is denoted by

$$
s_{1}(\beta) \leqq s_{2}(\beta)<s_{3}(\beta)<\cdots<s_{\nu}(\beta)<\ldots
$$

where $s_{1}(\beta)=s_{2}(\beta)$ if and only if $\beta=0$ and $\frac{1}{2}<\alpha<1$. $^{2}$

We denote by $A_{\nu}(\theta)$ the directed "empty" arc on the circle $K$ corresponding to $s_{\nu}(\beta)$, which does not contain $n \alpha$-points with $0<n<s . A_{\nu}(\beta)$ has positive or negative sign, according to the direction in which it starts from the point $\beta$. We denote the directed length of $\Delta_{\nu}(\beta)$ by $\delta_{v}(\beta)$ and the absclute length of it by $\overline{\boldsymbol{\delta}}_{\nu}(\beta)$.

If for an index $\nu$ the inequality $\delta_{v}(\beta) \delta_{\nu+1}(\beta)<0$ holds, we call this pair of adjacent multipla a pair of jumping multipla and denote it by $s_{v_{k}}(\beta)=q_{k}(\beta), s_{v_{k}^{\prime}+i}(\beta)=q_{k}^{\prime}(\beta) .{ }^{3}$ For the corresponding $\delta_{\nu}(\beta)$ we use the notation $\delta_{\nu_{k}}(\beta)=d_{k}(\beta), \delta_{v_{k}+1}(\beta)=d_{k}^{\prime}(\beta)$. For the sake of simplicity, in the case $\beta=0$ we use instead of $s_{\nu}(0), q_{k}(0)$ etc. only $s_{v}, q_{k}$ etc., respectively.

From the definition of the adjacent multipla $s_{v}(\beta)$ it follows that for an arbitrary positive integer $x \neq s_{\nu}(\beta)(\nu=1,2, \ldots)$ there is an $s_{v}(\beta)<x$ for which

and a fortiori

$$
\bar{\delta}_{r}(\beta)=\min _{y}\left|s_{\nu}(\beta) \alpha-\beta-y\right|<\min _{y}|x \alpha-\beta-y|
$$

$$
s_{\nu},\left(\beta, \bar{\delta}_{\nu}(\beta)<x|x \alpha-\beta-y| .\right.
$$

The homogeneous case. For the case $\beta=0$ we have proved in [3] and [4], as a simpic consequence of Property A, the following

${ }^{2}$ By this convention the recursive formulae in (2.2)-(2.4) are valid also for $k=1$.

${ }^{3}$ I. e., if the $s_{\gamma}(j) \alpha-$ and $s_{\gamma^{\prime}+1}(\beta) \alpha$-points approach the point $\beta$ from opposite sides. 
LEMMA I. If the $s_{\gamma} \alpha$-point is adjacent to $O$ and the $s_{\nu-l} \alpha$-point (l positive integer) among the $\alpha-, 2 a-, \ldots, s_{y} \alpha$-points is the nearest to $O$ from the opposite side, ${ }^{4}$ then

$$
\begin{aligned}
& s_{\nu+1}=s_{\nu}+s_{\nu-l}, \\
& \delta_{\nu+1}=\delta_{v}+\delta_{v^{\prime-l}} .
\end{aligned}
$$

Further, as a consequence of Lemma I and Property A, we have proved

LEMMA II. Let $a_{k}$ be defined $b y^{5}$

$$
a_{k}=\left[\left|\frac{d_{k-1}}{d_{k}}\right|\right]
$$

and let

$$
s_{0}=q_{0}=0, \quad d_{0}=\delta_{0}=-1, \quad q_{1}=q_{2} \text { if } s_{1}=s_{2},
$$

then for the above-defined quantities we have for $k==1,2, \ldots$

$$
\begin{array}{cl}
q_{k+1}=q_{k-1}+a_{k} q_{k}, \quad & d_{k+1}=d_{k-1}+a_{k} d_{k}, \quad \bar{d}_{k+1}=\bar{d}_{k-1}-a_{k} \bar{d}_{k}, \\
& q_{k+1} \bar{d}_{k}+q_{k} \bar{d}_{k+1}=1,
\end{array}
$$

and for $0<r<a_{k}$

$$
s_{v_{k}+r}=q_{k-1}+r q_{k}, \quad d_{v_{k}+r}=d_{k-1}+r d_{k}, \quad \bar{d}_{v_{k}+r}=\bar{d}_{k_{k-1}}-r \bar{d}_{k} .
$$

As a consequence of Lemma II it follows

LEMMA III. If $q_{k}<s_{\nu}<q_{k+1}$, then

These lemmas show that the above-defined multipla $q_{k}$ are identical with the convergents of $\alpha$, the other multipla $s_{v}$ with the by-denominators (Nebennenner) of $\boldsymbol{c}$. The numbers $a_{k}$ defined in (2.2) are identical with the digits of the continued fraction of $\alpha$.

From the definition of the multipla $q_{k}$ and from Lemmas I-II it follows the

REMARK. a) The sequence $d_{1}, \ldots, d_{k}, \ldots$ has alternative signs, $\left|d_{1}\right|, \ldots,\left|d_{k}\right|, \ldots$ is monotonically decreasing.

b) For an arbitrary $N$ among the $\alpha-, 2 \alpha_{-}, \ldots, N \alpha$-points the two adjacent points intercepting $O$ have always the form with a suitable $r$ and $k$.

$$
s_{\nu-l} \alpha=q_{k} \alpha, \quad s_{\nu} \alpha=\left(q_{k-1}+r q_{k}\right) \alpha \quad\left(0<r \leqq a_{k}\right) .
$$

c) The $\left(q_{k-1}+r q_{k}\right) \alpha$-points with $0<r \leqq \alpha_{k}$ are between the $q_{k-1} \alpha$-point and the point $O$, in the arc $A_{v_{k-1}}$, and with the restriction $n<q_{k+1}$ only these $n \alpha$-points are in $A_{v_{k-1}}$.

${ }^{4}$ I. e. $\delta_{\nu} \delta_{v-l}<0$ and in the arc $\Delta_{\nu}+\Delta_{\nu-l}$ there is no $n \alpha$-point with $0<n<s_{\nu}$.

${ }^{5}[x]$ denotes, as usual, the integral part of the real number $x$.

- 1. e. Lemma III diminishes further the set of those integer $x$ 's for which $x|x<-y|$ is the "least possible". 
The inhomogeneous case. Since in the case when $\beta=\langle n \alpha\rangle$ the approximation of $\beta$ by the multipla of $\alpha$ is similar to the case $\beta=0$, in the sequel we suppose that $\beta \neq\langle n \alpha\rangle$.

THEOREM 1. For every $0<\beta<1$ one can determine uniquely a sequence of integers $b_{1}, \ldots, b_{k}, \ldots$ with the property

$$
\begin{gathered}
1<b_{1} \leqq a_{1}+1, \quad 0 \leqq b_{k} \leqq a_{k} \quad(k=2,3, \ldots), \\
b_{k+1}=0 \text { only if } b_{k}=a_{k},
\end{gathered}
$$

so that the sequence of the adjacent multipla $s_{\nu}(\beta)$ is identical with the numbers

$$
b_{1} q_{1}+\cdots+b_{k-1} q_{k-1}+r q_{k}
$$

for $0<r \leqq b_{k}(k=1,2, \ldots)$.

PROof. In what follows we give a process for the determination of the numbers $b_{k}(k=1,2, \ldots)$ from which the statement of the theorem follows.

Determination of $b_{1}$. The $\alpha-, 2 \alpha-, \ldots,\left(a_{1}+1\right) \alpha$-points split the periphery of $K$ into $a_{1}+1$ disjunct arcs.

Case 1. If $\beta$ lies in the arc with length $\alpha=d_{1}$ bordered by the $(r-1) \alpha-$ and $r \alpha$-points with $0<r \leqq \alpha_{1}+1$, then let $b_{1}=r$.

Case 2. If $\beta$ is in the arc with length $\bar{d}_{2}$ bordered by the $\left(a_{1}+1\right) a$ and $\alpha$-points, then let $b_{1}=a_{1}+1$. From the definition of $b_{1}$ in both cases obviously follows that the $r \alpha$-points $\left(0<r \leqq b_{1}\right)$ - and with the restriction $n \leqq a_{1}+1$ only these - are adjacent to $p$.

In Case $1\left(b_{1}-1\right) \alpha$ and $b_{1} \alpha$ are jumping multipla, i. e. in this case

$$
q_{1}(\beta)=b_{1}-1=\left(b_{1}-1\right) q_{1}, \quad q_{1}^{\prime}(\beta)=b_{1}=b_{1} q_{1} .
$$

Next we determine $b_{k}$ supposing that $b_{1}, \ldots, b_{k-1}$ are already determined.

Case $k-1$.1. If $\beta$ is in an arc with length $\bar{d}_{k-1}$ bordered by the $\left(b_{1} q_{1}+\cdots+\left(b_{k-1}-1\right) q_{k-1}\right) \alpha-$ and $\left(b_{1} q_{1}+\cdots+b_{k-1} q_{k-1}\right) \alpha$-points, then we consider the points with multipla

$$
b_{1} q_{1}+\cdots+b_{k-1} q_{k-1}+r q_{k} \quad\left(0<r \leqq a_{k}\right) .
$$

According to Remark c) and Property A, these - and with the restriction $n \leqq b_{1} q_{1}+\cdots+b_{k-1} q_{k-1}+a_{k} q_{k}$ only these - points are in this arc with length $\bar{d}_{k-1}$. We determine $b_{k}$ in a similar way as $b_{1}$ by distinguishing two cases:

Case $k .1$. If the point $\beta$ is in one of the arcs with length $\vec{d}_{k}$ bordered by the $\left(b_{1} q_{1}+\cdots+b_{k-1} q_{k-1}+(r-1) q_{k}\right) \alpha-$ and $\left(b_{1} q_{1}+\cdots+b_{k-1} q_{k-1}+\right.$ $\left.+r q_{k}\right) \alpha$-points with $0<r \leqq a_{k}$, then let $b_{k}=r$.

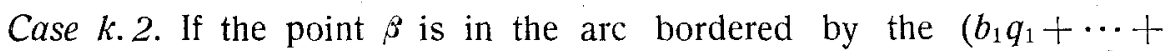
$\left.+b_{k-1} q_{k-1}+a_{k} q_{k}\right) \alpha-$ and $\left(b_{1} q_{1}+\cdots+\left(b_{k-1}-1\right) q_{k-1}\right) \alpha$-points with length $\bar{d}_{k-1}$, then let $b_{k}=a_{k}$. 
Case $k-1.2$. If $\beta$ is in the arc with length $\alpha_{k}$ bordered by the $\left(b_{1} q_{1}+\cdots+b_{k-2} q_{k-2}+a_{k-1} q_{k-1}\right) \alpha-\quad$ and $\quad\left(b_{1} q_{1}+\cdots+\left(b_{k-2}-1\right) q_{k-2}\right) \alpha-$ points, then let $b_{k}=0$. (Since the first point with smallest multiplum which lies in the arc bordered by these points, is the $\left(b_{1} q_{1}+\cdots+a_{k-1} q_{k-1}+q_{k-1}\right) \alpha$-point.)

From the definition of $b_{k}$ it follows, according to Remark c) and Property $\mathrm{A}$, that the $\left(b_{1} q_{1}+\cdots+b_{k-1} q_{k-1}+r q_{k}\right) \alpha$-points $\left(0<r \leqq b_{k}\right)-$ and with the restriction $b_{1} q_{1}+\cdots+b_{k-1} q_{k-1}<n<b_{1} q_{1}+\cdots+b_{k-1} q_{k-1}+a_{k} q_{k}$ only these - are adjacent to $\beta$.

COROLLARY. From the determination of the numbers $b_{k}$ it follows that if $b_{k+1} \neq 0$, then $s_{\nu}(\beta)=b_{1} q_{1}+\cdots+b_{k-1} q_{k-1}+\left(b_{k}-1\right) q_{k}$ and $s_{\nu+1}(\beta)=$ $=b_{1} q_{1}+\cdots+b_{k} q_{k}$ form a pair of jumping multipla, $i$. e. with a suitable $l$

$$
q_{i}(\beta)=b_{1} q_{1}+\cdots+\left(b_{k}-1\right) q_{k}, \quad q_{l}^{\prime}(\beta)=b_{1} q_{1}+\cdots+b_{k} q_{k} .
$$

If $b_{k+1}=0$, then it is suitable to call also the pair of multipla

$$
q_{t}(\beta) \equiv s_{\nu}(\beta)=b_{1} q_{1}+\cdots+b_{k} q_{k}, \quad q_{i}^{\prime}(\beta)=s_{\nu}(\beta)-q_{k+1}
$$

a pair of jumping multipla.

From Property A it follows that for every index $v$ the distance between the $s_{v}(\beta) \alpha$-point and the point $O$ has the form

$$
\left\langle s_{\nu}(\beta) \alpha\right\rangle=b_{1} d_{1}+\cdots+b_{k-1} d_{k-1}+r d_{k} \quad\left(0<r \leqq b_{k}\right) .
$$

Since the $n c$-points are everywhere dense on the periphery of $K$, the $\beta$-point is the limit of the $s_{v}(\beta) \alpha$-points, and so, according to $(2.10)$,

$$
\beta=\sum_{k=1}^{\infty} b_{k} d_{k} \text {. }
$$

From (2.10) and (2.11) we obtain for $\nu>\nu_{2}$

$$
\delta_{\nu}(\beta)=\left\langle s_{\nu}(\beta) \alpha\right\rangle-\beta=r d_{k}-\sum_{\nu=k}^{\infty} b_{\nu} d_{\nu} \circ
$$

The following theorem gives an analogous result as (2.5) for the inhomogeneous case :

Lemma IV. Let $b_{k+1} \neq 0, b_{k}>1,0<r<b_{k}-1$ and

(2.14) $s_{\nu}(\beta)=b_{1} q_{1}+\cdots+b_{k-1} q_{k-1}+\left(b_{k}-1\right) q_{k}\left(=q_{l}(\beta)\right), s_{\nu-r}(\beta)=s_{\nu}(\beta)-r q_{k}$;

- It follows directiy from the definition of the numbers $b_{k}$ that for every index $\nu$ $s_{v+1}(\beta)-s_{y}(\beta)=q_{k i}$ with a suitable $l$. Using this remark, $b_{k_{k}}$ could have been defined as the number of indices with the property $s_{\nu+1}(\beta)-s_{\nu}(\beta)=a_{r}$.

8 The difference between the treatment of CAssels-Descombes and that given above for the inhomogeneous case lies in the fact that the mentioned authors start from an arithmetical definition of the numbers $q_{k}(\beta)$ and deduce from it the minimum properlies, while we start from the minimum properties and deduce their arithmetical properties. 
then we have

(2. 15)

$$
s_{\nu}(\beta) \overline{\delta_{\nu}}(\beta)<S_{\nu-r}(\beta) \overline{\delta_{\nu \sim r}}(\beta) .
$$

If $b_{k+1}==0,\left(b_{k}=a_{k}\right)$, let $0<r<b_{k}$ and

$s_{\nu}(\beta)=b_{1} q_{1}+\cdots+b_{k-1} q_{k-1}+b_{k} q_{k}\left(=q_{l}(\beta)\right), \quad s_{\nu-r}(\beta)=s_{\nu}(\beta)-r q_{k}$,

then we have

$$
s_{\nu}(\beta) \bar{\delta}_{\nu}(\beta)<s_{\nu \rightarrow r}(\beta) \bar{\delta}_{\nu-r}(\beta) .
$$

PROOF. From the definition of the numbers $b_{k}$ it follows that $\delta_{\nu}(\beta) \delta_{y-r}(\beta)>0$ and, consequently, from $(2.13)$ and $(2.12)$

From this and (2.13)

$$
\bar{\delta}_{\nu}(\beta)=\bar{\delta}_{v-r}(\beta)-r \bar{d}_{k} \text {. }
$$

$$
\frac{s_{\nu \sim r}(\beta) \bar{\delta}_{\nu \sim r}(\beta)}{s_{\nu}(\beta) \bar{\delta}_{\nu}(\beta)}=\left(1-r \frac{q_{k}}{s_{\nu}(\beta)}\right)\left(1+r \frac{\bar{d}_{k}}{\overline{\delta_{\nu}}(\beta)}\right) .
$$

(2. 14) gives $s_{v^{\prime}}(\beta)>\left(b_{k}-1\right) q_{k}$. Further, from the definition of $b_{k}$ and $b_{k+1} \neq 0$ it follows that $\beta$ is in the arc with length $\vec{d}_{k}$ bordered by the $s_{y}(\beta) \alpha$ - and $\left(s_{v}(\beta)+q_{k}\right) \alpha$-points. Consequently,

$$
\overline{\delta_{v}}(\beta)<\overline{d_{k}} .
$$

Using these in (2.16), we obtain

$$
\frac{s_{\nu-r}(\beta) \bar{\delta}_{y-r}(\beta)}{s_{\nu}(\beta) \bar{\delta}_{\nu}(\beta)}>\left(1-\frac{r}{b_{k}-1}\right)(1+r) \geqq 1 .
$$

The proof of (2.15) runs analogously as the above proof of (2.14).

\section{§3}

For the proof of theorems of Borel type we need the following

LEMMA $V$. If $b_{k+1}=0$, then for

$$
q_{l}(\beta)=b_{1} q_{1}+\cdots+b_{k} q_{k}, \quad q_{i}(\beta)=q_{i}(\beta)-q_{k+1}
$$

we have

$$
\min \left(q_{l}(\beta) \bar{d}_{l}(\beta), q_{l}^{\prime}(\beta) \bar{d}_{l}^{\prime}(\beta)\right)<\frac{1}{3}{ }^{9}
$$

PROOF. Using the recursive formulae (2.3) and (2.7), we have

$$
q_{i}^{\prime}(\beta) \leqq\left(a_{1}+1\right) q_{1}+\cdots+a_{k} q_{k}=q_{k+1}+q_{k}, \quad q_{l}(\beta) \leqq q_{k} .
$$

From the definition of the numbers $b_{k}$ and $b_{k+1}=0$ it follows that $\beta$ is in the arc with length $\bar{d}_{k+1}$ bordered by the $q_{b}(\beta) \alpha$ - and $\left(q_{l}(\beta)-q_{k+1}\right) \alpha$-points.

9 'The same lemma occurs in Cassels' paper [1] 
Therefore

Let $t$ be defined by

$$
\bar{d}_{l}(\beta)+\bar{d}_{l}^{\prime}(\beta)=\bar{d}_{i+1}
$$

$$
\left(q_{k+1}+q_{k}\right) t=q_{k}\left(\bar{d}_{k-1}-t\right)
$$

Obviously, from (3.3) and (3.4), using also (2.4) we get

$$
\begin{aligned}
& \min \left(q_{l}(\beta) \bar{d}_{l}(\beta), q_{l}^{\prime}(\beta) \overline{d_{l}}(\beta)\right) \leqq \min \left(\left(q_{k+1}+q_{k}\right) \bar{d}_{l}(\beta), q_{k}\left(\bar{d}_{k+1}-\bar{d}_{l}(\beta)\right) \leqq\right. \\
& \leqq\left(q_{k+1}+q_{k}\right) t=\frac{q_{k+1}+q_{k}}{q_{k+1}+2 q_{k}} q_{k} \bar{d}_{k+1}<\frac{q_{k+1}+q_{k}}{q_{k+1}+2 q_{k}} \frac{q_{k}}{q_{k+1}} \frac{1}{\frac{q_{k}}{q_{k+1}}+\frac{\bar{d}_{k}}{\bar{d}_{k+1}}} .
\end{aligned}
$$

Since $0<\frac{q_{k}}{q_{k+1}}<1, \frac{\bar{d}_{k}}{\bar{d}_{k+1}}>1$, it follows that

$$
\min \left(q_{i}(\beta) \bar{d}_{l}(\beta), q_{l}^{\prime}(\beta) \overline{d_{l}^{\prime}}(\beta)\right)<\frac{q_{k}}{q_{k+1}} \frac{1}{1+2 \frac{q_{k}}{q_{k+1}}}<\frac{1}{3} .
$$

From this proof it is easy to see that the constant $\frac{1}{3}$ is best-possible.

THEOREM II. For every pair of jumping multipla we have

$$
\min \left(q_{l}(\beta) \bar{d}_{l}(\beta), q_{l}^{\prime}(\beta) \bar{d}_{l}^{\prime}(\beta)\right)<\frac{2}{3} .
$$

PROOF. Owing to Lemma $V$ we may suppose that the pair of jumping multipla $q_{l}(\beta), q_{i}^{\prime}(\beta)$ has the form as in (2.9). Similarly as in (3.3), we have

$$
q_{l}(\beta) \leqq q_{k+1}, \quad q_{i}^{\prime}(\beta) \leqq q_{k+1}+q_{k} .
$$

Similarly as we obtained (2.17), we have

$$
\bar{d}_{l}(\beta)+\bar{d}_{l}^{\prime}(\beta)=\bar{d}_{k} .
$$

Therefore, if $t$ is defined by

we have

$$
q_{k+1} t=\left(q_{k}+q_{k+1}\right)\left(\bar{d}_{k}-t\right)
$$

$$
\min \left(q_{l}(\beta) \bar{d}_{l}(\beta), q_{l}^{\prime}(\beta) \overline{d_{l}^{\prime}}(\beta)\right) \leqq q_{k+1} t=\frac{q_{k+1}+q_{k}}{2 q_{k+1}+q_{k}} q_{k+1} \bar{d}_{k} .
$$

From this, taking (2.4) and $\frac{q_{k}}{q_{k+1}}<1$ into account, it follows the statement of the theorem, and also the fact that the constant $\frac{2}{3}$ is best-possible.

THEOREM III. For any two consecutive pairs of jumping multipla the inequality holds.

$$
\min \left(q_{l}(\beta) \bar{d}_{l}(\beta), q_{l}^{\prime}(\beta) \bar{d}_{l}^{\prime}(\beta), \quad q_{l+1}(\beta) \bar{d}_{l+1}(\beta) ; q_{l+1}^{\prime}(\beta) \bar{d}_{l+1}^{\prime}(\beta)\right)<\frac{1}{2}
$$


By other words, the inequality $x|\alpha x-\beta-y|<\frac{1}{2}$ has a solution among any two consecutive pairs of jumping multipla.

Proof. Owing to Lemma $V$ we may suppose that both pairs of jumping multipla have the form as in (2.9), i. e.

$$
\begin{aligned}
q_{l}(\beta) & =b_{1} q_{1}+\cdots+b_{k-1} q_{k+1}+\left(b_{k}-1\right) q_{k}, & q_{l}^{\prime}(\beta) & =q_{l}(\beta)+q_{k}, \\
q_{l+1}(\beta) & =q_{l}^{\prime}(\beta)+\left(b_{k+1}-1\right) q_{k+1}, & q_{l+1}^{\prime}(\beta) & =q_{l}^{\prime}(\beta)+b_{k+1} q_{k+1} .
\end{aligned}
$$

Similarly as in (3.3), we have

$$
\begin{aligned}
q_{l}(\beta) & \leqq q_{k+1}, & q_{l}^{\prime}(\beta) & \leqq q_{k+1}+q_{k}, \\
q_{l+1}(\beta) & \leqq q_{k}+b_{k+1} q_{k+1}, & q_{i+1}^{\prime}(\beta) & \leqq q_{k}+\left(b_{k+1}+1\right) q_{k+1} .
\end{aligned}
$$
holds.

Let $u$ be defined by $\overline{d_{l+1}}(\beta)=u \overline{d_{k+1}}$. According to $(2.17)^{10} 0<u<1$

From (2.13), taking into account that the sequence $d_{1}, \ldots, d_{k}, \ldots$ has alternative signs, we have

$$
\begin{aligned}
\bar{d}_{l}(\beta) & =\bar{d}_{k}-\left(b_{k+1}-u\right) \bar{d}_{k+1}, & \bar{d}_{i}^{\prime}(\beta) & =\left(b_{k+1}-u\right) \bar{d}_{k+1}, \\
\bar{d}_{l+1}(\beta) & =(1-u) \bar{d}_{k+1}, & \bar{d}_{l+1}^{\prime}(\beta) & =u \bar{d}_{k+1} .
\end{aligned}
$$

With the notation $x=\frac{\bar{d}_{k}}{\bar{d}_{k+1}}, \quad y=\frac{\bar{q}_{k}}{\bar{q}_{k+1}}, \quad b=b_{k+1}$ we have from (3.6) and (3.7), using (2.4),

$$
\begin{aligned}
& q_{l}(\beta) \bar{d}_{k}(\beta) \leqq q_{k+1}\left(\bar{d}_{k}-\left(b_{k+1}-r\right) \bar{d}_{k+1}\right)=q_{k+1} \bar{d}_{k+1}\left(\frac{\bar{d}_{k:}}{\bar{d}_{k+1}}-\left(b_{k+1}-r\right)\right)= \\
& =\frac{1}{\frac{q_{k}}{q_{k+1}}+\frac{\bar{d}_{k}}{\bar{d}_{k+1}}}\left(\frac{\bar{d}_{k}}{\bar{d}_{k+1}}-\left(b_{k+1}-u\right)\right),
\end{aligned}
$$

$$
q_{i}(\beta) \bar{d}_{l}(\beta) \leqq \frac{1}{x+y}(x-b+u) \stackrel{\text { def }}{=} F_{1}(x, y, b, u) .
$$

Similarly

$$
\begin{aligned}
q_{l}^{\prime}(\beta) \bar{d}_{l}^{\prime}(\beta) & \leqq \frac{1}{x+y}(1+y)(b-u) \stackrel{\text { def }}{=} F_{2}(x, y, b, u), \\
q_{l+1}(\beta) \bar{d}_{l+1}(\beta) & \leqq \frac{1}{x+y}(b+y)(1-u) \stackrel{\text { def }}{=} F_{3}(x, y, b, u), \\
q_{l+1}^{\prime}(\beta) \bar{d}_{l+1}^{\prime}(\beta) & \leqq \frac{1}{x+y}(1+b+y) u \stackrel{\text { def }}{=} F_{4}(x, y, b, t u)
\end{aligned}
$$

$10 \beta$ is in the arc with length $\bar{d}_{k+1}$ bordered by the $q_{b+1}(\beta) \alpha$ - and $q_{l+1}^{\prime}(\beta) \alpha$-points. 
where

$$
\left(a_{k}<x<a_{k}+1, \quad 0<y<1, \quad 0<b \leqq a_{k}, \quad b \text { integer }\right) .
$$

Since from (3.12) we get $(1+y)(b-u) \geqq(b+y)(1-u)$ and consequently $F_{2} \geqq F_{3}$, for the proof of the theorem we have to show that with the restriction given in $(3.12)$ for $(x, y, b, u)$

$$
\sup _{x, y, b, u} \min \left(F_{1}, F_{3}, F_{4}\right) \leqq \frac{1}{2}
$$

holds. It follows obviously from (3.8)-(3.11) that if for a value of $(x, y, b, u$ ) for any pair $i \neq k$ also $F_{i} \neq F_{k}$, then one may change the value of $u$ so that $\min \left(F_{1}, F_{3}, F_{4}\right)$ increases. This is the fact also in the case when $F_{1}=F_{4}<F_{3}$. So for the determination of $\sup _{x, y, b, u} \min \left(F_{1}, F_{3}, F_{4}\right)$ we have to investigate the following two cases only:

1. $F_{1}==F_{3} \leqq F_{4}$,

2. $F_{3}=F_{4} \leqq F_{1}$.

Case 1. From $F_{1}=F_{3}$ it follows $x-b+r=(y+b)(1+r)$,

$$
r=\frac{y+2 b-x}{y+b+1}, \quad 1-r=\frac{x-b+1}{y+b+1},
$$

and consequently in this case

$$
\text { (3. 13) } \quad F_{1}=F_{3}=\frac{1}{x+y}(b+y) \frac{x-b+1}{y+b+1}, \quad F_{4}=\frac{1}{x+y}(1+b+y) \frac{y+2 b-x}{1+b+y}
$$

and, since $F_{1}=F_{3} \leqq F_{4}$, therefore $(b+y)(x-b+1) \leqq(1+b+y)(y+2 b+x)$, i. e.

$$
x \leqq \frac{(y+b)^{2}}{2 y+2 b+1}+b
$$

From $b \geqq 1$ it follows that

$$
F_{1}=F_{3}=\frac{x-b+1}{x+y} \frac{b+y}{1+b+y}=\left(1-\frac{y+b-1}{x+y}\right) \frac{b+y}{1+b+y}
$$

is monotonically increasing in $x$. Putting therefore in (3.13) the upper bound of $x$, from (3.14), we get

$$
F_{1}=F_{3} \leqq \frac{y+b+1}{3 y+3 b+1} \leqq \frac{1}{2}
$$

In Case 2 we can obtain in a quite analogous way the same expression of $y$ and $b$ as an upper bound for the minimum in question. These: together prove the theorem. 
From the proof it follows that for the values

$$
\begin{aligned}
& b=b_{k+1}=1, \\
& y=\frac{q_{k}}{q_{k+1}} \sim 0 \quad\left(a_{k} \text { large }\right), \\
& x=\frac{\bar{d}_{k}}{\bar{d}_{k+1}} \sim \frac{4}{3} \quad\left(a_{k+1}=1, \quad a_{k+2}=3, a_{k+3} \text { large }\right), \\
& u=\frac{1}{3}
\end{aligned}
$$

we have $F_{1}=F_{3}=F_{4} \sim \frac{1}{2}$, i. e. the constant $\frac{1}{2}$ is best-possible.

THEOREM IV. For every positive integer $l$ there exists a suitable irrational $\alpha$ and $a$ real $\beta \neq\langle n \alpha\rangle$ so that with arbitrary $\varepsilon>0$ for an infinity of $k_{0}$ the inequality

holds.

$$
\min _{k_{0}<k<k_{0}+i}\left(q_{k}(\beta) \bar{d}_{k}(\beta), \quad q_{k}^{\prime}(\beta) \bar{d}_{k}^{\prime}(\beta)\right)>\frac{1}{\sqrt{5}}-\varepsilon
$$

By other words, the inequality $x|\alpha x-\beta-y|<\frac{1}{\sqrt{5}}-\varepsilon$ cannot be satisfied among the $l$ consecutive pairs of jumping multipla and, according to Lemma IV, also among the numbers $x$ with $q_{k_{0}}(\beta)<x<q_{k_{0}+l}^{\prime}(\beta)$.

Proof. The theorem follows easily from the following

LEMMA. Let \& be an arbitrary small positive number and the integer $M$ sufficiently large. If for an $\alpha$ and $\beta$ and for $k-2 M \leqq v \leqq k+2 M(k>2 M)$. we have

$$
a_{v}=b_{v}=1,
$$

then putting

the inequality

$$
q_{k}(\beta)=q_{k-1}^{\prime}(\beta)=b_{1} q_{1}+\cdots+b_{k-1} q_{k-1},
$$

holds.

$$
q_{k}(\beta) \bar{d}_{k}(\beta)>\frac{1}{\sqrt{5}}-\varepsilon
$$

PROOF OF THE LEMma. Using the recursive formulae (2.3), we obtain according to (3.16)

$$
\begin{aligned}
& \text { (3. 17) } q_{k}(\beta)=b_{1} q_{1}+\cdots+b_{k-1} q_{k-1}<\sum_{\nu=k-2 M}^{k-1} b_{\nu} q_{\nu}=\sum_{\nu=k-2 M}^{k-1} q_{\nu}=\sum_{\nu=k-2 M}^{k-1} a_{\nu} q_{v}= \\
& =\sum_{v=k_{k-2 M}}^{k-1}\left(q_{v+1}-q_{\nu-1}\right)=q_{k}+q_{k-1}-q_{k-2 M}-q_{k-2 M-1}=q_{k+1}-q_{2 M+1}>q_{k+1}\left(1-\varepsilon^{\prime}\right)
\end{aligned}
$$

where $\varepsilon^{\prime}$ is arbitrary small if $M$ is large enough. 
Similarly, for $\bar{d}_{k}(\beta)$ we have from $(2.3)$ and $(3.16)$

Since

$$
d_{k}(\dot{0})=d_{k}+\sum_{\nu=k+1}^{\infty} b_{\nu} d_{\nu}=\sum_{\nu=k}^{k+2 M-1} d_{v}+\sum_{v=k+2 M}^{\infty} b_{\nu} d_{\nu}
$$

$$
\begin{gathered}
\sum_{\nu=k}^{k+2 M-1} d_{\nu}=\sum_{\nu=k}^{k+2 M-1} a_{\nu} d_{v}=\sum_{\nu=k}^{k+2 M-1}\left(d_{\nu+1}-d_{\nu-1}\right)=-d_{k-1}-d_{k}+d_{2 M-1}+d_{2 M}= \\
=-d_{k+1}+d_{2 M+1}
\end{gathered}
$$

and

we have

$$
\left|\sum_{\nu=k+2 M}^{\infty} b_{\nu} d_{\nu}\right| \leqq \sum_{\nu=0}^{\infty} a_{k+2 M+2 \nu} \bar{d}_{k+2 M+2 \gamma^{\prime}}==\bar{d}_{k+2 M-1}
$$

$$
\bar{d}_{k}(\beta)>\bar{d}_{k+1}-\bar{d}_{k+2 M-1}-\bar{d}_{k+2 M}>\bar{d}_{k+1}\left(1-\varepsilon^{\prime \prime}\right)
$$

where $\varepsilon^{\prime \prime}$ is arbitrary small if $M$ is large enough.

From (3.17) and (3.18) we get

$$
q_{k}(\beta) \bar{d}_{k}(\beta)>q_{k+1} \bar{d}_{k+1}\left(1-\varepsilon^{\prime}\right)\left(1-\varepsilon^{\prime \prime}\right)=\frac{1}{\frac{q_{k}}{q_{k+1}}+\frac{\bar{d}_{k}}{\bar{d}_{k+1}}}\left(1-\varepsilon^{\prime}\right)\left(1-\varepsilon^{\prime \prime}\right) \text {. }
$$

Since the first $2 M$ digits of the continued fractions of $\frac{q_{k}}{q_{k+1}}$ and $\frac{\bar{d}_{k i}}{\bar{d}_{k+1}}$ are the same as those for the numbers

$$
\frac{\sqrt{5}-1}{2}=\frac{1}{1+} \frac{1}{1+} \cdots \text { and } 1+\frac{\sqrt{5}-1}{2}=1+\frac{1}{1+\frac{1}{1+\cdots}}
$$

respectively, we obtain

$$
\frac{1}{\frac{q_{k}}{q_{k+1}}+\frac{\overline{\bar{d}}_{k}}{\overline{\bar{d}}_{k+1}}}>\frac{1}{\sqrt{5}-1}+1+\frac{\sqrt{5}-1}{2}\left(1-\varepsilon^{\prime \prime \prime}\right)=\frac{1}{\sqrt{5}}\left(1-\varepsilon^{\prime \prime \prime}\right) .
$$

From (3. 19) the Lemma follows if $M$ is so large that

$$
\left(1-\varepsilon^{\prime}\right)\left(1-\varepsilon^{\prime \prime}\right)\left(1-\varepsilon^{\prime \prime \prime}\right)>(1-\varepsilon) \text {. }
$$

Theorem IV is a simple consequence of this. Namely, if in the sequence $a_{k}(k=1,2, \ldots)$ we have for an infinity of indices $v$

$$
a_{\nu}=a_{\nu^{\prime}+1}=\cdots=a_{\nu+2 M+l}=1 \text {, }
$$

this means that the conditions of the Lemma are fulfilled for $l$ consecutive indices $k_{0}+1, \ldots, k_{0}+l$ and for infinitely many $k_{0}$. Further, if for infinitely many $\mu$-indices $a_{\mu} \neq 1$ and $b_{\mu} \neq a_{\mu}, b_{\mu} \neq 0$, then, as one can see from the definition of the numbers $b_{k}, \beta \neq\langle n \alpha\rangle$. 


\section{Bibliography}

[1] J. W. S. Cassels, On a theorem of Khintchine, Proc. London Math. Soc. (2), 53 (1951), pp. $310-320$.

[2] R. Descombes, Sur la répartition des sommets d'une ligne polygonale regulière nonfermée, Annales scientifiques d'école normale supérieure, 75 (1956), pp. 284--355.

[3] Vera T. Sós, On the theory of diophantine approximations. I, Acta Math. Acad. Sci. Hung., 8 (1957), pp. 461-472.

[4] Vera T. Sós, A lánctörtek egy geometriai interpretációja és alkalmazásai, Math. Lapok, 8 (1957), pp. 248-263.

[5] Vera T. Sós, On a theorem of A. Khintchine, Acta Arithmetica (under press).

[6] Vera T. Sós, On the theory of diophantine approximations. III, to appear in Acta Math. Acad. Sci. Hung. 